\title{
Phosphoglycerate Mutase Family
}

National Cancer Institute

\section{Source}

National Cancer Institute. Phosphoglycerate Mutase Family. NCI Thesaurus. Code C123808.

A family of proteins whose members are involved in glycolysis. This family is comprised of three isozymes that are either homodimers or heterodimers of phosphoglycerate mutase 1 and 2 . These enzymes have multiple reversible isomerase and phosphatase activities. During glycolysis these enzymes can interconvert 3-phosphoglycerate to 2phosphoglycerate through a 2,3-bisphosphoglycerate intermediate. 